\title{
Is it time to revise the diagnostic criteria for apathy in brain disorders? The 2018 international consensus group
}

\author{
P. Robert ${ }^{\mathrm{a}, *, 1}$, K.L. Lanctôt ${ }^{\mathrm{b}, 1}$, L. Agüera-Ortiz ${ }^{\mathrm{c}}$, P. Aalten ${ }^{\mathrm{d}}$, F. Bremond $^{\mathrm{a}, \mathrm{e}}$, M. Defrancesco ${ }^{\mathrm{f}}$,

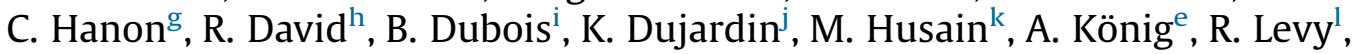 \\ V. Mantua ${ }^{\mathrm{m}}$, D. Meulien ${ }^{\mathrm{n}}$, D. Miller ${ }^{\mathrm{O}}$, H.J. Moebius ${ }^{\mathrm{p}}$, J. Rasmussen ${ }^{\mathrm{q}}$, G. Robert ${ }^{\mathrm{r}}$, \\ M. Ruthirakuhan ${ }^{\mathrm{b}}$, F. Stella ${ }^{\mathrm{s}}$, J. Yesavage $^{\mathrm{t}}$, R. Zeghari $^{\mathrm{a}}$, V. Manera $^{\mathrm{a}, \mathrm{e}}$
}

a CoBTeK IA, Memory Centre, University Cote d'Azur, France

${ }^{\mathrm{b}}$ Sunnybrook Research Institute and Departments of Pharmacology/Toxicology and Psychiatry, University of Toronto, Toronto, Canada

'Department of Psychiatry, Instituto de Investigacio'n Sanitaria (imas12), Hospital Universitario 12 de Octubre E' Centro de Investigacio'n Biome'dica en Red de Salud Mental (CIBERSAM), Madrid, Spain

d School for Mental Health and Neuroscience, Alzheimer Center Limburg, Maastricht, Netherlands

e STARS team - INRIA Sophia Antipolis, Valbonne 06902, France

${ }_{\mathrm{f}}$ Department of Psychiatry and Psychotherapy, Innsbruck Medical University, Austria

${ }^{\mathrm{g}}$ Regional Resource Centre of Old Age Psychiatry, Paris Descartes University, Assistance Publique-Hôpitaux de Paris, Corentin-Celton Hospital, Issy-lesMoulineaux, France

h Memory Center CMRR, CHU - CoBTeK lab, University Cote d'Azur, France

${ }^{i}$ Centre des Maladies Cognitives et Comportementales (IM2A), Institut du Cerveau et de la Moelle épinière (ICM), UMR-S975, AP-HP, Salpêtrière Hospital, Sorbonne University, Paris, France

j Neurology and Movement Disorders, Lille University Medical Center, Inserm U1171, Lille, France

${ }^{\mathrm{k}}$ Nuffield Department of Clinical Neurosciences (John Radcliffe Hospital, Oxford OX3 9DU) E Department of Experimental Psychology, University of Oxford, UK

${ }^{1}$ AP-HP, Neurology deparment, Salpetriere hospital Inserm, U 1127, CNRS, UMR 7225, Sorbonne Universités, UPMC Univ Paris 06, UMR S 1127, Institut du

Cerveau et de la Moelle épinière, ICM, FRONTlab School of medicine, Sorbonne Universités, Paris, France

m Agenzia Italiana del Farmaco, via del Tritone 181, 00187, Rome, Italy

${ }^{n}$ Clinical Research Neurology Lundbeck SAS, Denmark

o Bracket, Wayne, PA, USA

P moebius-consult GmbH, Baar, ZG, Switzerland

${ }^{\mathrm{q}}$ psi-napse, Surrey, UK

rEA4712 "Comportement et noyaux gris centraux", Université de Rennes 1, France

s Laboratorio de Neurociencias LIM27, Departamento e Instituto de Psiquiatria, Hospital das Clinicas HCFMUSP, Faculdade de Medicina, Universidade de Sao

Paulo, UNESP - Univ Estadual Paulista, Biosciences Institute, Campus of Rio Claro, SP, Brazil

${ }^{\mathrm{t}}$ Department of Psychiatry and Behavioral Sciences, Stanford University, School of Medicine, Stanford University, Stanford, CA, USA

\section{A R T I C L E I N F O}

\section{Article history:}

Received 21 April 2018

Received in revised form 19 July 2018

Accepted 24 July 2018

Available online 17 August 2018

\section{Keywords:}

Apathy

Neuropsychiatry

Brain disorders

Motivation

Behaviour

Emotion

Social interaction

Treatment

\begin{abstract}
A B S T R A C T
Background: Apathy is a very common behavioural and psychological symptom across brain disorders. In the last decade, there have been considerable advances in research on apathy and motivation. It is thus important to revise the apathy diagnostic criteria published in 2009. The main objectives were to: a) revise the definition of apathy; b) update the list of apathy dimensions; c) operationalise the diagnostic criteria; and d) suggest appropriate assessment tools including new technologies.

Methods: The expert panel $(\mathrm{N}=23)$ included researchers and health care professionals working on brain disorders and apathy, a representative of a regulatory body, and a representative of the pharmaceutical industry. The revised diagnostic criteria for apathy were developed in a two-step process. First, following the standard Delphi methodology, the experts were asked to answer questions via web-survey in two rounds. Second, all the collected information was discussed on the occasion of the 26th European Congress of Psychiatry held in Nice (France).

Results: Apathy was defined as a quantitative reduction of goal-directed activity in comparison to the patient's previous level of functioning (criterion A). Symptoms must persist for at least four weeks, and affect at least two of the three apathy dimensions (behaviour/cognition; emotion; social interaction;
\end{abstract}

\footnotetext{
* Corresponding author at: CMRR Institut Claude Pompidou, 10 rue Molière, 06100 Nice, France.

E-mail address: probert@unice.fr (P. Robert).

${ }^{1}$ Equal contribution.
} 
criterion B). Apathy should cause identifiable functional impairments (criterion C), and should not be fully explained by other factors, such as effects of a substance or major changes in the patient's environment (Criterion D).

Conclusions: The new diagnostic criteria for apathy provide a clinical and scientific framework to increase the validity of apathy as a clinical construct. This should also help to pave the path for apathy in brain disorders to be an interventional target.

(c) 2018 The Author(s). Published by Elsevier Masson SAS. This is an open access article under the CC BYNC-ND license (http://creativecommons.org/licenses/by-nc-nd/4.0/).

\section{Introduction}

Apathy is a pervasive neuropsychiatric symptom of most neurocognitive, neurodegenerative, and psychiatric disorders. It represents the most common behavioural and psychological symptom in people with Alzheimer's disease [1] and Huntington's disease [2], and is prevalent in other neurodegenerative conditions, such as Parkinson's disease [3] and vascular dementia [4]. It is also found among substantial proportions of individuals following stroke and traumatic brain injury [5], and psychiatric conditions such as major depressive disorder [6] and schizophrenia [7]. The presence of apathy significantly affects the patient's quality of life [8], and in neurodegenerative disorders is associated with a faster cognitive and functional decline [9], representing a risk factor for the conversion from Mild Cognitive Impairment to Alzheimer's disease [10]. For all these reasons, identifying apathy early in disease progression is considered a clinical and research priority.

In major contributions [11-14], apathy was defined as a lack of motivation that persists over time and causes identifiable functional impairment. Three dimensions of apathy were identified, including deficits in goal-directed behaviour, goal-directed cognitive activity, and emotions. In 2008, a task force was set up during the European Psychiatric Association congress to develop diagnostic criteria for apathy [15]. Based on these diagnostic criteria, a patient is classified as apathetic when he/she meets four criteria (A-D). Criterion A specifies the presence of a loss of (or diminished) motivation in comparison to the person's previous level of functioning, which is not consistent with his age or culture. These changes in motivation may be reported by the patient himself or by the observations of others. Criterion B stipulates the presence of symptoms in at least two of three domains (behaviour, cognition, and emotion) for a period of at least four weeks and present most of the time. These symptoms can be detected either in self-initiated or environment-stimulated activities. Criterion $C$ specifies that the symptoms (A - B) must cause clinically significant impairment in personal, social, occupational domains, or other important areas of functioning. Finally, Criterion D specifies that the symptoms (A - B) should not exclusively explained or due to physical or motor disabilities, to diminished level of consciousness or to the direct physiological effects of a substance.

These diagnostic criteria for apathy are now widely used in clinical and research practice for patients with neurodegenerative and neuropsychiatric disorders (e.g., [16]).

In the last decade, there have been considerable advances in the domain of apathy in brain disorders, including the apathy biological and neural based (e.g., [17]). First, the definition of apathy as a disorder of 'motivation' (Criterion A) has been extensively criticized (e.g. [18],), as 'motivation' is a psychological interpretation of behavioural internal states, which may be difficult to measure objectively. At the same time, the construct of goal directed behaviour/activity - construed as a set of related processes by which an internal state is translated, through observable action, into the attainment of a goal (e.g., [19]) - is increasingly used in the domain of neuroscience, and it has been proposed to be a useful to operationalize apathy, particularly in clinical contexts. Second, the different apathy domains (criterion B) have been object of discussion, and most particularly: a) the distinction between the 'behaviour' and 'cognition' domains and its relevance in clinical practice [20]; b) the importance of adding the 'social interaction' as a domain of apathy [21]; c) the importance of considering alternative proposals on apathy subtypes based on the underlying disrupted mechanisms (for instance, the 'emotional-affective', 'cognitive' and 'auto-activation' apathy subtypes [18]. Third, finer assessment tools for apathy have been developed, based on classical instruments (e.g., interviews and self-reports; see [17] for a review) but also on new Information and Communication Technologies (ICTs, e.g., [22]). However, no consensus has been reached so far on the role of ICTs in the apathy assessment, and on their relations to classical apathy measures.

Finally, the therapeutic strategy is an important aspect to consider. Despite the lack of an established pharmacological treatment for apathy with a strong evidence base (e.g., [23]), preliminary data on apathy treatment efficacy are emerging [24], with a research focus on drugs [25] and repetitive transcranial magnetic stimulation [26], often accompanied by non-pharmacological approaches [27]. Having diagnostic criteria for apathy based on the last advancements in the clinical research that reach a wide consensus among the scientific, regulatory and medical community is therefore crucial. These would, for example, allow clinical trials to be designed with a well-defined population and more sensitive apathy outcome measures, and thus obtain wider acceptance regarding the effectiveness of prevention and/or treatment strategies.

Given all these advances, a group of experts in the domain of apathy in brain disorders (leaded by PR and $\mathrm{KL}$ ) decided to revise the diagnostic criteria for apathy proposed in 2008. The main objectives were to: a) revise the definition of apathy (criterion $A$ ); b) update the list of apathy dimensions (criterion B); c) operationalize the diagnostic criteria using examples of clinical situations and areas of possible impairment (criterion B); and d) suggest appropriate and updated apathy assessment tools.

\section{Methods}

\subsection{Task force}

Participants were selected based on their expertise in the domain of apathy in brain disorders. Some of these experts already participated to the 2008 expert meeting. These included, among others, clinicians and researchers from a) the CoBTeK-IA lab and Memory centre of the University Côte d'Azur, a lab with a focus on how to assess apathy using ICT); b) the French Memory Centre network, which includes 17 research memory centres located in the French university hospitals, c) the ISCTM (International Society for CNS Clinical Trials and Methodology) Apathy Workgroup; d) the ISTAART (International Society to Advance Alzheimer's Research and Treatment) Neuropsychiatric symptoms professional Interest Area.

The final task force included 23 experts (researchers, health care professionals and representatives of one regulatory body and 
of the pharmaceutical industry). The complete list of the experts, together with their field of expertise, is reported in STable 1.

Following the standard Delphi methodology [28], the revised diagnostic criteria for apathy were developed in a two-step process: web-surveys followed by a consensus meeting.

\subsection{Web-surveys}

The experts were asked to answer questions via web-survey in two rounds (between November 2017 and February 2018). After each round, a facilitator (PR) provided a summary of the experts' responses, and encouraged the experts to analyze, comment and (eventually) revise their earlier responses in light of the commentaries of other members of the panel. The questions for the second round were designed according to the responses obtained in the first round. In addition, between the first and second round, the experts were asked to work in subgroups in order to focus on different topics (generate definitions, find relevant examples in the clinical practice, indicate the potential to employ new technologies). After the second round, a first draft of the new criteria, including the elements of the two rounds and the documents provided by the subgroups, were circulated among all the experts. A shortened version of the questionnaire (questions 1 to 4) was also sent to the French Memory Centers network, which includes 17 research memory centers located in the French university hospital. Responses were obtained from 11 centers (64\%).

\subsection{Final consensus meeting}

The two web-survey results and the open discussion points were revised by the task force during the $26^{\text {th }}$ European Congress of Psychiatry held on March 5, 2018 in Nice (France). A consensus was reached when all participants agreed on the decision to modify (or not) specific aspects of the current diagnostic criteria for apathy.

\section{Results}

The new proposed diagnostic criteria for apathy, to be employed both in the clinical and the research domain, are reported in Table 1 and in the Appendix (Supplementary Materials). The list of questions and responses for the two websurveys are reported in STable 2 (responses obtained from 17 participants).

\subsection{Title}

Diagnostic Criteria for Apathy. No modification was performed to be consistent with the previous literature.

\subsection{Criterion $A$}

This stipulates the presence of quantitative reduction of goaldirected activity either in the behavioural, cognitive, emotional or social dimension in comparison to the patient's previous level of functioning. These changes may be reported by the patient him/ herself or by the observation of others.

Modifications: 1) The term 'motivation' was replaced by 'goaldirected behaviour/activity', which is easier to observe and objectively quantify (see [18]). 2) The sentence 'which is not consistent with his/her age or culture' was removed, as it was considered unnecessary (as apathy is defined as a reduction compared to the person's previous level of functioning); 3 ) the

Table 1

Apathy diagnostic criteria 2018.

CRITERION A: A quantitative reduction of goal-directed activity either in behavioral, cognitive, emotional or social dimensions in comparison to the patient's previous level of functioning in these areas. These changes may be reported by the patient himself/herself or by observation of others.

CRITERION B: The presence of at least 2 of the 3 following dimensions for a period of at least four weeks and present most of the time B1. BEHAVIOUR \& COGNITION

Loss of, or diminished, goal-directed behaviour or cognitive activity as evidenced by at least one of the following:

General level of activity: the patient has a reduced level of activity either at home or work, makes less effort to initiate or accomplish tasks spontaneously, or needs to be prompted to perform them.

Persistence of activity: $\mathrm{He} /$ she is less persistent in maintaining an activity or conversation, finding solutions to problems or thinking of alternative ways to accomplish them if they become difficult.

Making choices: He/she has less interest or takes longer to make choices when different alternatives exist (e.g., selecting TV programs, preparing meals, choosing from a menu, etc.)

Interest in external issue: $\mathrm{He} / \mathrm{she}$ has less interest in or reacts less to news, either good or bad, or has less interest in doing new things

Personal wellbeing: He/she is less interested in his/her own health and wellbeing or personal image (general appearance, grooming, clothes, etc.). B2. EMOTION

Loss of, or diminished, emotion as evidenced by at least one of the following:

Spontaneous emotions: the patient shows less spontaneous (self-generated) emotions regarding their own affairs, or appears less interested in events that should matter to him/her or to people that he/she knows well.

Emotional reactions to environment: He/she expresses less emotional reaction in response to positive or negative events in his/her environment that affect him/her or people he/she knows well (e.g., when things go well or bad, responding to jokes, or events on a TV program or a movie, or when disturbed or prompted to do things he/she would prefer not to do).

Impact on others: He/she is less concerned about the impact of his/her actions or feelings on the people around him/her.

Empathy: He/she shows less empathy to the emotions or feelings of others (e.g., becoming happy or sad when someone is happy or sad, or being moved when others need help).

Verbal or physical expressions: He/she shows less verbal or physical reactions that reveal his/her emotional states.

B3. SOCIAL INTERACTION Loss of, or diminished engagement in social interaction as evidenced by at least one of the following:

Spontaneous social initiative: the patient takes less initiative in spontaneously proposing social or leisure activities to family or others.

Environmentally stimulated social interaction: He/she participates less, or is less comfortable or more indifferent to social or leisure activities suggested by people around him/her.

Relationship with family members: He/she shows less interest in family members (e.g., to know what is happening to them, to meet them or make arrangements to contact them).

Verbal interaction: $\mathrm{He} / \mathrm{she}$ is less likely to initiate a conversation, or he/she withdraws soon from it

Homebound: He /She prefer to stays at home more frequently or longer than usual and shows less interest in getting out to meet people.

CRITERION C These symptoms (A - B) cause clinically significant impairment in personal, social, occupational, or other important areas of functioning.

CRITERION D The symptoms (A - B) are not exclusively explained or due to physical disabilities (e.g. blindness and loss of hearing), to motor disabilities, to a diminished level of consciousness, to the direct physiological effects of a substance (e.g. drug of abuse, medication), or to major changes in the patient's environment. 
different apathy dimensions were listed in the definition (the behavioural, cognitive, emotional or social dimensions), to specify immediately the relevant domains.

\subsection{Criterion $B$}

This indicates that apathy is a persistent state, rather than a transient or intermittent one by incorporating in the definition a minimum duration of four weeks [29]. It also stipulates that symptoms should be observed in at least 2 out of the following 3 dimensions:

\subsubsection{Behaviour $\mathcal{E}$ cognition}

This includes the loss of, or diminished, goal-directed behaviour and cognitive activity as evidenced by at least one of 5 examples / situations. This domain embeds the loss of, or diminished, goaldirected behaviour, i.e., a reduction in routine (habitual) and nonroutine (occasional) activities, and a reduced goal-directed cognitive activity, usually interpreted in practical terms as a loss of, or diminished, interests.

\subsubsection{Emotion}

This included the loss of, or diminished, emotion as evidenced by at least one of 5 examples/situations including both spontaneous emotions, and emotions in response to the environment/ others.

\subsubsection{Social interaction}

This refers to the loss of, or diminished, engagement in social interaction as evidenced by at least one of 5 examples/situations. These include both spontaneous social initiative, and environment/others stimulated social interaction.

Modifications: 1) the term 'domains' was replaced with 'dimensions', as there is some overlap between behaviour/ cognition, emotion and social interaction areas. 2) The apathy dimensions were modified (previous dimensions: Cognition; Behavior; Emotion). First, as deficits in 'Behavior' and 'Cognition' were found to be frequently associated in the clinical practice and in large studies (e.g., [20]) it was agreed that these two dimensions could be included in a single category. Furthermore, it was agreed that it is important to add the 'Social interaction' dimension. The fact that impaired social interaction is an important domain of apathy has been previously highlighted by Sockeel and colleagues during the development of the LARS scale [29]. There is now converging evidence that the social dimension may represent a separate apathy domain, distinct from cognition/behaviour and emotion [30-33, 34]. 3). The difference between self-initiated and environmentally stimulated deficits was presented in the examples provided for each dimension, instead of being part of the main definition. 4) Areas of impairment (five examples per dimension) were added. This was considered as important to help clinicians in their everyday practice, and to help to operationalize the diagnostic criteria. The examples were drafted by a sub-group of experts based on their clinical experience, and validated by all the experts task force.

\subsection{Criterion $C$}

This refers to the presence of clinically significant functional impairment (e.g., in personal, social and/or occupational domains) largely attributable to the symptoms specified in criteria A and B. No modification was performed.

\subsection{Criterion $D$}

The intent is to exclude from the definition conditions and states that might not be distinguished from apathy solely on the basis of the criteria above (e.g., diminished level of consciousness), as well as transient states of apathy that can be attributed to a discrete non-neuropsychiatric cause (e.g., direct physiological effects of a drug/medication). This criterion also suggests taking into account whether major changes in the patient's environment occurred (e.g., severe conflicts, loss of significant people), that might completely explain the observed symptoms.

Modifications: The following sentence was added "The symptoms (A-B) are not exclusively explained or due to major changes in the patient's environment". This was decided after acknowledging that major environmental events, such as a terrorist attack, may lead to major changes in several aspects of everyday life that may mimic apathy (e.g., social isolation, emotional blunting, reduction of activities outside).

\subsection{Appendices}

The appendices (available online as Supplementary Materials as well as in the criteria at the following link: http://www. innovation-alzheimer.fr/wp-content/uploads/2018/08/ApathyCriteria-2018.pdf includes the following elements:

1 A definition of Goal-directed behaviour/activity: This section defines goal-directed behaviour/activity as behaviour aimed toward a goal or toward the completion of a task. It also considers the presumed pathophysiological mechanisms underpinning apathy as being the consequence of various underlying dysfunctions of mental and biological processes required to elaborate, initiate and control intentional/goaldirected behaviour).

2 A summary of the main instruments that can be employed to assess apathy. Specifically, apathy can be assessed through:

a) A number of clinical scales and indexes (e.g., [16]), based on the patient's self-reports, and/or informant-based scales and indexes, and/or clinician's scales. STable 3 reports the selected clinical scales/indexes available, specifying whether they include a patient, informant and/or clinician version; the apathy dimensions that each instrument assesses, and the population for which the instrument was initially designed/ tested.

b) New Information and Communication Technologies (ICT). There is emerging evidence that new ICT approaches could provide clinicians with valuable additional information in terms of assessment, and therefore more accurate diagnosis of apathy (e.g., [22]). ICT instruments that could be employed to assess different aspects and dimensions of apathy include: actigraphy and methods used to monitor motor activity and rest-activity rhythms (e.g., [35]); voice analysis, video analysis, serious games, and robots, which are already developed, but currently employed only in research settings (e.g. [36],).

Some cautions were also added. For instance, regarding clinical scales, it was reported that, due to anosognosia, patient's reports should be taken with caution. Regarding the caregiver's reports, it is important to select a reliable caregiver (e.g., someone who spends sufficient time with the patient, and is able to provide an accurate evaluation) as well as taking into account their potential biases. It is possible to use other types of scales / assessment tools in parallel to assess co-morbidities (e.g., for depression, anxiety, fatigue). Concerning ICT, results should be interpreted with caution in patients with movement disorders who may show poverty of movement in the absence of apathy (e.g., Parkinson's disease, Huntington's disease, progressive supranuclear palsy). These patients often have reduced total activity, related to their motor symptoms. Likewise, they may speak slowly, with a hypophonic 
voice, have low speech rates due to speech and respiratory deficits; or have a hypomimic face that can give the erroneous impression that they do not have emotional reactions.

3 A summary of what is needed for pharmacological clinical trials. This includes providing: a) the scientific rationale (biological basis) for targeting specific dimensions; b) the relation with the product intended for development (mechanism of action); c) justification for the selection of clinical trial endpoints (see discussion).

\section{Discussion}

Recent literature points to the importance of adopting a trans diagnostic approach, which cuts across traditional disease boundaries, to provide useful means for better understanding of apathy and related conditions [37]. Reaching a wide, international consensus on the definition and dimensions of apathy is a crucial step in this direction. The expert panel that worked to develop the 2018 diagnostic criteria for apathy involved clinicians working on different brain disorders, including Alzheimer's disease and other neurodegenerative disorders, major depression and schizophrenia. Similarly, it involved people from different disciplines, such as adult and geriatric psychiatry, neurology, neuropsychopharmacology, informatics and technology as well as people from the pharmaceutical industry and from the regulatory domain. For this reason, we are convinced that the 2018 apathy criteria may have an impact on the clinical and research community.

The 2018 apathy diagnostic criteria keep the same overall structure (criteria A-D) compared to the previous criteria [15]. However major modifications were performed. Specifically:

Criterion A: the term motivation was replaced by goal-directed behavior. This was a pragmatic choice, because 'goal-directed behaviors' are easier to observe and describe compared to motivation, which is an internal state that can only be inferred from behaviour observation. This is also in line with the recent scientific literature [18].

Criterion B: the expert panel faced the challenge of keeping the same 3-dimentions structures. This is for pragmatic reasons, as the diagnostic criteria should be easy and fast to assess in the clinical practice. At the same time, it was important to include a broader spectrum of symptoms, in particular related to the social interaction aspects. This led to add 'social interaction' as a separate dimension (B3), and for practical and theoretical reasons, to subsume behavioral and cognitive domains of apathy under one category (B1). This association could be criticised because the category 'behaviorcognition' is very broad, and puts together symptoms that can potentially be dissociated based on the underling brain processes (e.g., $[38,39]$ ). However, in the clinical practice, it is difficult to dissociate cognitive from behavioral deficits, because both result in diminished observable activity. The revision of the diagnostic criteria is not primarily meant for basic research purposes; it is mainly meant to improve the clinical description, and thus the assessment of apathy across brain disorders in the domain of clinical research and the everyday clinical practice. In this domain the association between 'behavior' and 'cognition' makes sense. The clinical focus also explains why the dimensions included in the present criteria do not totally overlap with the sub-forms of apathy described by Levy and Dubois [18], that is the 'emotionalaffective, cognitive' and 'auto-activation' subtypes. That classification relies on potential mechanisms underlying apathy, while the classification presented here refers to the dimensions for which impairment can be observed at the clinical level. The focus on designing more understandable and easy to use criteria also justifies the efforts done for operationalizing each domain with the same number of examples. In the case of the B1 dimension, these include both cognitive and behavioral aspects. Furthermore, the examples systematically include symptoms related to activity selfinitiation, and to activities in response to the environment stimulation.

The validation of these criteria in clinical practice will determine if this 3-dimension structure is meaningful, and which are the most frequent examples found for each dimension.

As in the previous diagnostic systems, the presence of impairment in at least two dimensions is needed to fulfill criterion B. This allows the possibility that some patients with a deficit in a single apathy dimension are not classified as apathetic. It is important to keep track of these single-dimension deficits, most particularly for the early pathologies stages, in order to better understand if there is a continuum between single symptoms and apparition of a full apathy spectrum. This is of interest in the field of $\mathrm{AD}$ and related disorders, but also in other neuropsychiatric diseases.

In parallel to facilitate the ease of use in the clinical practice, one of the major challenges of the new criteria is to pave the path for the use of new technologies in the assessment domain. This is true for apathy but also for other neuropsychiatric symptoms. The assessment methods will improve and become more objective thanks to ICT. This is why the present apathy criteria provide symptoms descriptions as objective as possible, and encompassing the overall spectrum of symptoms. Today, despite some limitations, the motor activity assessment seems the most robust method, as reported in the Appendix found in theSupplementary Materials (e.g., [40,41]). But, in the next future, emerging technologies such as audio and video sensors could be part of the clinicians' choice options (e.g. [36],).

The new criteria should also provide additional elements to inform the choice of non-pharmacological treatments targeted to a specific patient. For instance, examples were included for each dimension to help to indicate if the symptoms concerned spontaneous behavior, or the behavior in response to other people or the environment.

We are convinced that the 2018 apathy diagnostic criteria may also be important to provide more objective and internationally recognized criteria for apathy assessment for pharmacological therapeutic research. Despite the lack of biological or molecular understanding of many of the phenomenological domains in the context of major psychiatric or neurological categories, regulatory science is moving towards a dimensional approach to drug targets. Phenomenological domains can be described within or across diagnostic entities. In order to accept a phenomenological domain as a drug target, a rationale should be provided for the validity of its construct and the value for the patients. The new diagnostic criteria for apathy provide a clinical and scientific framework to increase the validity and the value of apathy as a clinical construct. Since the present revision of the diagnostic criteria considers apathy as a domain that exists across clinical diagnoses such as major and mild neurocognitive disorders, schizophrenia and major depression, the consistency of the evaluation should be demonstrated independently from the population in which the assessment is carried out. As a general regulatory requirement, clinical trials designed to test a specific hypothesis of efficacy on apathy in the context of a specific diagnostic category are required, as well as adequate endpoints. Also, depending on whether apathy is targeted in the context of dementia, or schizophrenia or major depression, the relationship with the time course of the underlying pathology should be established. Adequate justification why the product has the potential to improve apathy will be needed. 


\section{Acknowledgments and authors' note}

The expert meeting and the Delphi panel were supported by the Edmond \& Lily Safra Foundation, Institute Claude Pompidou, University Côte d'Azur, and by the IA association. Thanks for the ISTCM and ISTAART contribution, and a special acknowledgment to all clinicians and researchers working for so many years on the topic. The apathy diagnostic is part of the MNC3 program University Cote d'Azur.

Valentine Mantua: The opinions expressed in this manuscript are the personal views of the Author and may not be understood or quoted as being made on behalf of or reflecting the position of the Italian (AIFA) or European (EMA) Medicines Agency or any of their Committees. The mention of commercial products, their sources, or their use in connection with material reported herein is not to be constructed as either an actual or implied endorsement of such products by any Public Department or Health and/or Payer Services.

\section{Appendix A. Supplementary data}

Supplementary data associated with this article can be found, in the online version, at https://doi.org/10.1016/j.eurpsy.2018.07.008.

\section{References}

[1] Zhao Q., Tan L, Wang H, Jiang T, Tan M, Tan L, et al. The prevalence of neuropsychiatric symptoms in Alzheimer's disease: systematic review and meta-analysis. J Affect Disord 2016;190:264-71.

[2] Craufurd D, Thompson J, Snowden J. Behavioral changes in huntington disease. Cogn Behav Neurol 2001;14(4):219-26.

[3] den Brok MGHE, van Dalen JW, van Gool WA, et al. Apathy in Parkinson's disease: a systematic review and meta-analysis. Mov Disord 2015;30(May (6)):759-69.

[4] Staekenborg S, Su T, van Straaten E, Lane R, Scheltens P, et al. Behavioural and psychological symptoms in vascular dementia; differences between smalland large-vessel disease. J Neurol Neurosurg Psychiatr 2009;81(5):547-51.

[5] Starkstein S, Pahissa J. Apathy following traumatic brain injury. Psychiatr Clin North Am 2014;37(1):103-12.

[6] Yuen G, Bhutani S, Lucas B, Gunning F, AbdelMalak B, Seirup J, et al. Apathy in late-life depression: common, persistent, and disabling. Am J Geriatr Psychiatry 2015;23(5):488-94.

[7] Yazbek H, Norton J, Capdevielle D, Larue A, Boulenger J, Gély-Nargeot M, et al. The lille apathy rating scale (LARS): exploring its psychometric properties in schizophrenia. Schizophr Res 2014;157(1-3):278-84.

[8] Yeager C, Hyer L. Apathy in dementia: relations with depression, functional competence, and quality of life. Psychol Rep 2008;102(3):718-22.

[9] Starkstein S, Jorge R, Misrahi R, Robinson R. A prospective longitudinal study of apathy in Alzheimer's disease. J Neurol Neurosurg Psychiatr 2006;77(1):8-11.

[10] Papastavrou E, Kalokerinou A, Papacostas SS, Tsangari H, Sourtzi P. Caring for a relative with dementia: family caregiver burden. J Adv Nurs 2007;58(June (5)):446-57.

[11] van Reekum R, Stuss D, Ostrander L. Apathy: Why Care? J Neuropsychiatry Clin Neurosci 2005;17(1):7-19.

[12] Marin R. Differential diagnosis of apathy and related disorders of diminished motivation. Psychiatr Ann 1997;27(1):30-3.

[13] Marin R. Apathy: a neuropsychiatric syndrome. J Neuropsychiatry Clin Neurosci 1991;3(3):243-54.

[14] Starkstein S, Petracca G, Chemerinski E, Kremer J. Syndromic Validity of Apathy in Alzheimer's disease. Am J Psychiatry 2001;158(6):872-7.

[15] Robert P, Onyike C, Leentjens A, Dujardin K, Aalten P, Starkstein S, et al. Proposed diagnostic criteria for apathy in Alzheimer's disease and other neuropsychiatric disorders. Eur Psychiatry 2009;24(2):98-104.

[16] Radakovic R, Harley C, Abrahams S, Starr J. A systematic review of the validity and reliability of apathy scales in neurodegenerative conditions. Int Psychogeriatr 2015;27(6):903-23.
[17] Le Heron C, Apps M, Husain M. The anatomy of apathy: A neurocognitive framework for amotivated behaviour. Neuropsychologia 2017.

[18] Levy R, Dubois B. Apathy and the functional anatomy of the prefrontal cortexbasal ganglia circuits. Cereb Cortex 2005;16(7):916-28.

[19] Brown R, Pluck G. Negative symptoms: the 'pathology' of motivation and goaldirected behaviour. Trends Neurosci 2000;23(9):412-7.

[20] Mulin E, Leone E, Dujardin K, Delliaux M, Leentjens A, Nobili F, et al. Diagnostic criteria for apathy in clinical practice. Int J Geriatr Psychiatry 2011;26(2):15865.

[21] Ang Y, Lockwood P, Apps M, Muhammed K, Husain M. Distinct subtypes of apathy revealed by the apathy motivation index. PLoS One 2017;12(1) e0169938.

[22] König A, Aalten P, Verhey F, Bensadoun G, Petit P, Robert P, et al. A review of current information and communication technologies: can they be used to assess apathy? Int J Geriatr Psychiatry 2013;29(4):345-58.

[23] Feldman HH, Ferris S, Winblad B, Sfikas N, Mancione L, He Y, et al. Effect of rivastigmine on delay to diagnosis of Alzheimer's disease from mild cognitive impairment: the InDDEx study. Lancet Neurol 2007;6(June (6)):501-12.

[24] Mitchell RA, Herrmann N, Lanctôt KL. The role of dopamine in symptoms and treatment of apathy in Alzheimer's disease. CNS Neurosci Ther 2011;17 (October (15)):411-27.

[25] Scherer RW, Drye L, Mintzer J, Lanctôt K, Rosenberg P, Herrmann N, et al. ADMET 2 Research Group. The Apathy in Dementia Methylphenidate Trial 2 (ADMET 2): study protocol for a randomized controlled trial. Trials 2018;19 (1):46.

[26] Padala PR, Padala KP, Lensing SY, Jackson AN, Hunter CR, Parkes CM, et al. Repetitive transcranial magnetic stimulation for apathy in mild cognitive impairment: A double-blind, randomized, sham-controlled, cross-over pilot study. Psychiatry Res 2018;261:312-8.

[27] Kales HC, Gitlin LN, Lyketsos CG. Assessment and management of behavioral and psychological symptoms of dementia. BMJ 2015;350:h369.

[28] Linstone HA, Turoff M. The delphi method: techniques and applications. Reading, MA: Addison-Wesley; 1975.

[29] Sockeel P, Dujardin K, Devos D, Deneve C, Destée A, Defebvre L. The Lille apathy rating scale (LARS), a new instrument for detecting and quantifying apathy: validation in Parkinson's disease. J Neurol Neurosurg Psychiatr 2006;77 (5):579-84.

[30] Chang SWC, Fagan NA, Toda K, Utevsky AV, Pearson JM, Platt ML, et al. Neural mechanisms of social decision-making in the primate amygdala. PNAS 2015;112:.

[31] Starkstein SE, Leentjens AF. The nosological position of apathy in clinical practice. J Neurol Neurosurg Psychiatry 2008;79(10):1088-92.

[32] Lockwood PL, Hamonet M, Zhang SH, Ratnavel A, Salmony FU, Husain M, et al. Prosocial apathy for helping others when effort is required. Nat Hum Behav 2017;1(7).

[33] Apps MAJ, Rushworth MFS, Chang SWC. The anterior cingulate gyrus and social cognition: tracking the motivation of others. Neuron 2016;90(4):692-707.

[34] Lockwood P, Hamonet M, Zhang S, Ratnavel S, Salmony F, Husain M, et al Prosocial apathy for helping others when effort is required. Nat Hum Behav 2017;1:0131.

[35] David R, Mulin E, Friedman L, Le Duff F, Cygankiewicz E, Deschaux O, et al. Decreased daytime motor activity associated with apathy in Alzheimer disease: an actigraphic study. Am J Geriatr Psychiatry 2012;20(September (9)):806-14.

[36] König A, Satt A, Sorin A, Hoory R, Toledo-Ronen O, et al. Automatic speech analysis for the assessment of patients with predementia and Alzheimer's disease. Alzheimer's Demen: Diagn Assess Dis Monit 2015;1(1):112-24.

[37] Husain M, Roiser JP. Neuroscience of apathy and anhedonia: a transdiagnostic approach. Nature Reviews Neuroscience 2018;19:470-84.

[38] Stella F, Radanovic M, Aprahamian A, Canineu PR, de Andrade LP, Forlenza OV. Neurobiological correlates of apathy in Alzheimer's Disease and mild cognitive impairment: A critical review. Journal of Alzheimer's Disease 2014;39:633-48.

[39] Benoit M, Clairet S, Koulibaly PM, Darcourt J, Robert PH. Brain perfusion correlates of the apathy inventory dimensions of Alzheimer's disease. Int J Geriatr Psychiatry 2004;19:864-9.

[40] Yakhia M, Konig A, van der Flier WM, Friedman L, Robert PH, David R. Actigraphic motor activity in mild cognitive impairment patients carrying out short functional activity tasks: comparison between mild cognitive impairment with and without depressive symptoms. J Alzheimers Dis 2014;40 (4):869-75.

[41] Zeitzer JM, David R, Friedman L, et al. Phenotyping apathy in individuals with Alzheimer disease using functional principal component analysis. Am J Geriatr Psychiatry 2013;21(4):391-7. 